\title{
O MANEJO DA DOR EM CRIANÇAS COM CÂNCER: CONTRIBUIÇÕES PARA A ENFERMAGEM
}

\author{
Patrícia Curti Bueno'; Eliane Tatsch Neves²; Angelita Gastaldo Rigon ${ }^{3}$
}

\begin{abstract}
RESUMO: Pesquisa qualitativa descritiva retrospectiva objetivou descrever como a Enfermagem maneja a dor na criança com câncer. Os dados foram coletados nos prontuários de crianças internadas em uma unidade especializada no tratamento do câncer de um hospital de ensino do Sul do Brasil entre maio e junho de 2008. Os dados quantitativos foram submetidos à análise descritiva e os registros de enfermagem foram organizados em um quadro sinóptico ao qual foi aplicada a análise temática. Os resultados mostraram que $47,27 \%$ das crianças apresentavam queixas de dor. O tratamento medicamentoso foi o principal para toda e qualquer manifestação de choro; métodos menos invasivos e/ou estratégias alternativas de manejo foram raros. Conclui-se que há necessidade de se estabelecer métodos de avaliação e manejo da dor, conforme a faixa etária e o nível de desenvolvimento da criança e realizar registros de enfermagem com atenção, aliando o conhecimento técnico-científico à sensibilidade e afetividade.
\end{abstract}

PALAVRAS-CHAVE: Enfermagem pediátrica; Neoplasias; Dor; Registros de enfermagem.

\section{PAIN MANAGEMENT IN CHILDREN WITH CANCER: CONTRIBUTIONS TO NURSING}

\begin{abstract}
This qualitative, descriptive, retrospective research aimed to describe how Nursing manages pain in children with cancer. The data were collected from the hospital notes of children hospitalised in a unit specialising in the treatment of cancer in a teaching hospital in South Brazil between May and June 2008. The quantitative data were submitted to descriptive analysis and the nursing notes were organised in a synoptic chart to which thematic analysis was applied. The results showed that $47.27 \%$ of children presented complaints of pain. Treatment with medicines was the principal response to all and any manifestations of crying; less invasive, or alternative strategies of pain management were rare. It was concluded that there is a need to establish methods for assessment and management of pain, according to the age group and level of development of the child, and to keep nursing notes with attention, allying technical-scientific knowledge with sensitivity and affection.
\end{abstract}

KEYWORDS: Pediatric nursing; Neoplasia; Pain; Nursing notes.

\section{EL MANEJO DEL DOLOR EN NIÑOS CON CÁNCER: CONTRIBUCIONES PARA A ENFERMERÍA}

RESUMEN: Investigación cualitativa descriptiva retrospectiva cuyo objetivo fue describir como la Enfermería maneja el dolor en niño con cáncer. Los datos fueron recogidos en los prontuarios de los niños internados en una unidad especializada en tratamiento de cáncer de un hospital de enseñanza del Sur de Brasil entre mayo y junio de 2008. Los datos cuantitativos fueron sometidos al análisis descriptivo y los registros de enfermería fueron organizados en un cuadro sinóptico al cual fue aplicado el análisis temático. Los resultados revelaron que $47,27 \%$ de los niños presentaban quejas de dolor. El tratamiento medicamentoso fue el principal para toda y cualquier manifestación de lloro; métodos menos invasivos y/o estrategias alternativas de manejo fueron raros. Se concluye que hay necesidad de establecerse métodos de evaluación y manejo del dolor, de acuerdo a la franja etaria y el nivel de desarrollo del niño y realizar registros de enfermería con antención, uniendo el conocimiento técnico-científico a la sensibilidad y afectividad. PALABRAS CLAVE: Enfermería pediátrica; Neoplasías; Dolor; Registros de enfermería.

${ }^{1}$ Enfermeira. Especialista em Sistema Público de Saúde. Membro do Grupo de Pesquisa Cuidado à Saúde das Pessoas, Famílias e Sociedade.

${ }^{2}$ Enfermeira Pediatra. Doutora em Enfermagem. Professora do Departamento e do Programa de Pós-Graduação em Enfermagem da UFSM. Membro do Grupo de Pesquisa Cuidado à Saúde das Pessoas, Famílias e Sociedade.

${ }^{3}$ Enfermeira. Mestre em Enfermagem . Membro do Grupo de Pesquisa Cuidado à Saúde das Pessoas, Famílias e Sociedade.

Autor correspondente:

Angelita Gastaldo Rigon

Universidade Federal de Santa Maria

Rua Dona Luíza, 123 - 97010-160 - Santa Maria-RS-Brasil.

Recebido: 22/12/10

E-mail: enfangel@ibest.com.br

Aprovado: 09/03/11

Cogitare Enferm. 2011 Abr/Jun; 16(2):226-31 


\section{INTRODUÇÃO}

Em todo o mundo, milhões de pessoas morrem anualmente devido ao câncer, os casos dobraram entre 1975 e 2000, e devem duplicar novamente entre 2000 e 2020. Em 2030 o câncer poderá matar 17 milhões de pessoas, contra os 7,6 milhões de óbitos que provocou em 2007. Já entre a população infantil, o câncer pediátrico representa entre $0,5 \%$ e $3 \%$ de todos os tumores na maioria das populações ${ }^{(1)}$.

No Brasil o número de casos de câncer tem crescido, atingindo, em média, entre 12 e 13 mil crianças anualmente. $\mathrm{O}$ aumento de sua incidência é resultado das transformações globais das últimas décadas, da situação de saúde dos povos alterada pela urbanização acelerada, dos modos de vida e padrões de consumo ${ }^{(2)}$.

Em contrapartida, desde 1970, vem-se observando um aumento linear das taxas de cura dos tumores na infância, estando estas, atualmente, entre 70\% e 90\% dos casos nos Estados Unidos. No Brasil, as crianças e jovens com leucemia, por exemplo, curam-se em $70 \%$ a $80 \%$ dos casos $^{(3)}$. No entanto, o inimigo maior do câncer infantil é o diagnóstico tardio. Conforme estudos realizados em 2006 pela Fundação Centro de Controle Oncologia, entre $60 \%$ e $70 \%$ das crianças que chegavam à Fundação estavam em estágio avançado da doença, o que diminui enormemente as chances de cura $^{(4)}$.

Com isso, percebe-se que a demanda de crianças portadoras de neoplasias nos hospitais é expressiva e tende ao crescimento. Para além dos avanços na área de oncopediatria, a equipe de saúde ainda se depara com situações desafiantes durante o processo de enfrentamento da doença. Dentre estes desafios, salienta-se a dor no câncer infantil, que necessita de diagnóstico e intervenção adequados.

A dor em crianças está presente em $25 \%$ das consultas ambulatoriais, $50 \%$ das consultas hospitalares e em $80 \%$ dos procedimentos terapêuticos e diagnósti$\cos ^{(5)}$. A Agência Americana de Pesquisa e Qualidade em Saúde Pública e a Sociedade Americana de Dor a descrevem como o quinto sinal vital, e como tal, deve ser avaliado, tão automaticamente quanto os outros sinais vitais do paciente. Nesse sentido, acredita-se que as intervenções para o alívio da dor devem fazer parte do cuidado de enfermagem. Avaliar a dor e empenhar medidas para seu alívio, proporcionando conforto e bem-estar ao sujeito, podem ser considerados como dispositivos capazes de promoção da saúde durante a internação hospitalar ${ }^{(6)}$. Muitas vezes, a dor pode ser considerada mais angustiante e incapacitante do que qualquer doença isolada ${ }^{(7)}$.

A equipe de enfermagem convive intensamente com a clientela infantil e seu julgamento profissional é de extrema importância para o manejo adequado do sintoma dor. Se a dor própria não é fácil de compreender, a dor do outro é mais complicada ainda, principalmente em crianças, as quais nem sempre conseguem se expressar de forma objetiva.

Nesse sentido, compreende-se que o conhecimento sobre dor, e seu manejo, necessitam estar em constante atualização para acompanhar os avanços tecnológicos e contribuir com os cuidados de enfermagem.

Consultando a literatura científica pertinente à temática, constatou-se que esse assunto tem sido objeto de várias pesquisas. Entretanto, observou-se lacunas no que se refere à importância atribuída ao manejo da dor em crianças, que, muitas vezes, é subestimado por profissionais de saúde que convivem com a presença da dor como sintoma de inúmeras patologias.

Nesse sentido, questiona-se: como a equipe de enfermagem tem atuado no manejo da dor na criança com câncer? Para investigar essa questão, objetivou-se descrever como a Enfermagem tem manejado a dor na criança com câncer hospitalizada.

\section{METODOLOGIA}

Trata-se de uma pesquisa documental do tipo descritiva e retrospectiva. O cenário do estudo foi uma unidade especializada em tratamento do câncer de um hospital de ensino de referência no atendimento em oncologia pediátrica para toda a Macro-região Centro-Oeste do Estado do Rio Grande do Sul.

$\mathrm{O}$ estudo seguiu as recomendações constantes da Resolução 196/96 do Conselho Nacional de Saúde e o projeto de pesquisa foi aprovado pelo Comitê de Ética em Pesquisa da Universidade Federal de Santa Maria sob número 0046.0.243.000-08.

Os sujeitos foram selecionados tendo como critérios de inclusão: idade entre zero e 12 anos e internação no ano de 2006. Assim, os sujeitos do estudo foram 55 crianças entre as 437 internações ocorridas naquele ano. A maioria dos prontuários das crianças contava com múltiplos períodos de internação e os dados analisados correspondem às informações da última internação do período do estudo.

A coleta dos dados foi realizada por meio de um formulário próprio, testado previamente, que incluía variáveis relacionadas às queixas e à frequência de 
dor e manejo adotado para o seu alívio pela equipe de enfermagem e constantes nos registros de enfermagem, obtidos nos prontuários.

Os dados quantitativos foram submetidos à análise descritiva quantitativa, em frequências absoluta e relativa, e os registros de enfermagem foram organizados em um quadro analítico ao qual foi aplicada a análise de conteúdo temática ${ }^{(8)}$.

\section{RESULTADOS}

Das crianças que internaram em 2006 na unidade estudada, 33 (60\%) encontravam-se em idade escolar, entre seis e 12 anos; já as crianças em idade pré-escolar, de três e cinco anos foram 13 (24\%) e 9 lactentes (16\%).

No que se refere ao sexo, o número de meninos foi de $35(63,63 \%)$ e o de meninas foi de $20(36,36 \%)$.

Os tipos de cânceres que esteviveram em maior evidência entre as crianças estudadas foram as leucemias, o Linfoma não-Hodgkin, a Púrpura e o Linfoma de Hodgkin.

A maioria da população estudada ficou internada até cinco dias, no máximo, sendo um total de 30 crianças (55\%); já 11 crianças (20\%) estiveram internadas entre 6 e 10 dias, também 11 crianças (20\%) ficaram internadas por mais de 15 dias. A minoria das crianças, três ao todo $(5 \%)$, ficou internadas por um período de 11 a 15 dias.

Os registros de enfermagem apontaram a mãe como principal cuidadora, na maior parte do tempo de internação, para 45 crianças (81\%), o pai para $8(15 \%)$ e a tia ou a avó somaram $4 \%$.

O manejo da dor na criança com câncer foi analisado de acordo com os registros de enfermagem constantes em seus prontuários. Entre as 55 crianças, 26 referiram dor, representando um percentual de $47,27 \%$; 17 delas eram do sexo masculino e 9 do sexo feminino, o que em percentagem nos diz que foram $65,38 \%$ dos meninos que relataram dor em comparação com $34,61 \%$ das meninas. Dentre a população masculina houve um total de 64 registros de dor, em um universo de 17 meninos entre os 35 estudados. Já na população feminina, o número de registro chegou a 52, num universo de 9 meninas que apresentaram queixa de dor entre as 20 estudadas.

A queixa mais comum entre os meninos foi a cefaleia, seguida por dor articular, abdominal e dor no peito. Um fator importante na análise dos dados foi a quantidade de queixas da população masculina sem registro que indicasse sua localização. Dos 64 registros,
29 não indicaram o local, tendo apenas a descrição de "criança chorosa", "criança gemente" e "chorando copiosamente".

Já entre a população feminina houve maior queixa de dor lombar, seguida de dor abdominal e cefaleia. Nesta população, os casos de local da dor não identificado aconteceram em menor proporção, sendo 15 de um total de 52 registros de dor.

Os diagnósticos das 26 crianças que tiveram queixas de dor incluíram leucemias, linfoma não-Hodgkin, anemia e neuroblastoma. Destas doenças, as leucemias e o neuroblastoma foram os diagnósticos envolvidos no maior número de queixas de dor, tendo a primeira 54 registros e a segunda 28 registros.

As queixas de dor nos pacientes com leucemia apresentaram-se em grande número, representando $46,55 \%$ do total de registros. Essas características prevalecem nos dados dos registros de dor, sendo que dos 54 registros nas crianças com leucemia, 14 foram queixas relacionadas às articulações, cinco de cefaleia e 21 registros apenas indicavam que a criança chorava muito e/ou tinha expressão de dor, fazendo-se necessário o uso de analgésico para acalmá-la, sem especificar o local exato da dor ou mesmo o seu tipo.

Nos 116 registros de dor que surgiram durante a internação das crianças, o uso de medicamento foi a escolha predominante pela equipe de enfermagem responsável. As três medicações mais usadas para amenizar a dor foram: paracetamol em 49 registros (42,24\%), morfina em 29 registros (25\%) e codeína em 8 registros $(6,9 \%)$, totalizando 87 registros de manejo de dor utilizando-se medicamentos, com um total de $75 \%$ dos 116 registros encontrados.

Constatou-se, também, que os mesmos analgésicos eram usados nas crianças com diferentes tipos de queixa e diferente intensidade da dor, algumas vezes identificadas pelas palavras: "choro compulsivo"; "gemente" e "chorosa".

Observou-se, ainda, que os registros médicos e de enfermagem sobre quais critérios seriam adotados para a administração do analgésico não constavam nos prontuários e nem se foram aplicados para a avaliação da dor.

Ressalta-se que por diversas vezes durante a análise dos dados, a falta do registro da conduta foi frequente, deixando sem resposta o questionamento se foi, ou não, realizada alguma conduta para o manejo da dor.

Outro aspecto observado refere-se ao registro da administração da medicação, sem, contudo, especificála, impossibilitando a identificação da droga utilizada 
e da sua resolutividade.

Em relação ao manejo da dor por meio de estratégias não medicamentosas, identificou-se apenas quatro registros de conduta, sendo elas: o colo da mãe, a troca de decúbito, o uso bolsa de água quente e a mamadeira.

\section{DISCUSSÃO}

Os resultados convergem com a literatura quando apontam as crianças em idade escolar como as mais frequentes dentre a clientela pediátrica em tratamento contra o câncer ${ }^{(9)}$. Segundo o Instituto Nacional do Câncer, o câncer pediátrico representa entre $0,5 \%$ e $3 \%$ de todas as neoplasias na maioria das populações. Em geral, a incidência total de tumores malignos na infância é maior no sexo masculino ${ }^{(3)}$.

Desde 1970, observa-se um aumento progressivo, e também linear, das taxas de incidência dos tumores em crianças, os tumores do sistema nervoso central, os linfomas não-Hodgkin, o tumor de Wilms e outros tumores renais. Por exemplo, de 10 a 15 casos de câncer, entre indivíduos com menos de 15 anos de idade, no Brasil, quatro são de LLA, resultando em uma porcentagem de aproximadamente $30 \%{ }^{(3)}$, no que este estudo veio corroborar.

Vale ressaltar que o hospital do cenário deste estudo é referência para toda a região central do Estado do Rio Grande do Sul, tendo em vista a alta complexidade e a eficácia no tratamento, dispondo de uma equipe especializada em Hematologia.

O tempo de internação depende da fase do tratamento em que se encontra a criança, sendo que nos casos de leucemias, por exemplo, normalmente a primeira internação é bastante longa, pois inclui o tempo de estadiamento e de indução. Em fase de manutenção do tratamento, as drogas utilizadas são menos agressivas e o estado geral do paciente normalmente é melhor, o que diminui seu tempo de internação.

A mãe, ou outro familiar do sexo feminino, tem sido apontada como cuidadora principal de crianças e essa situação é explicada tendo por base as questões sócio-culturais do cuidado feminino ${ }^{(10)}$.

As queixas de dor foram mais reincidentes entre as meninas que em relação aos meninos, porém um maior número de meninos apresentou queixa de dor em relação às meninas. Quase a metade das crianças teve queixas de dor, o que vem reforçar a importância da atenção a este quinto sinal vital ${ }^{(6,11)}$. Em crianças, a dor é influenciada e determinada pelo seu nível de desenvolvimento, pela maneira como a criança comunica a sua dor e sua habilidade para enfrentá-la. Ou seja, a expressão da subjetividade da criança está intimamente relacionada à sua idade e sua maturidade cognitiva, $\mathrm{o}$ que leva à reflexão da necessidade de compreensão e entendimento da equipe que assiste a criança quanto às características e formas de expressão $0^{(11)}$.

A leucemia é uma doença maligna dos glóbulos brancos e de origem, na maioria das vezes, não conhecida. Um dos sintomas clínicos relativamente frequente é a dor óssea (articulações, membros inferiores e superiores), indicando o comprometimento leucêmico do periósteo e da $\operatorname{cortical}^{(9)}$. Há que se levar em consideração que as leucemias possuem um tratamento bastante agressivo e que, tanto este como seus efeitos colaterais, também ocasionam dor.

Para o alívio da dor, a farmacoterapia analgésica baseia-se em uma sequência terapêutica que se tornou conhecida como "escada analgésica", onde a orientação na manipulação da dor inicia por analgésico comum e/ ou antiinflamatórios não hormonais e as drogas adjuvantes. Caso este passo não resolva a dor, associa-se opioides fracos para, posteriormente, associar opioide forte ${ }^{(4)}$. Os dados do estudo apontaram o uso do paracetamol como droga analgésica comum, pois não atua sobre a medula óssea e suas células. Foi utilizado como droga de escolha para alívio da dor e parece ter sido resolutivo em muitos casos. Ocorre, no entanto, que a proporção entre o uso de codeína (opioide fraco associado ao analgésico antes do uso do opioide forte) e o da morfina (opioide forte) tem um sentido inverso (usou-se mais morfina que codeína), o que sugere o não-seguimento da farmacoterapia analgésica e falta de padronização de conduta.

Nesse sentido, estudos defendem que, na maioria dos hospitais, é comum observar prescrições como rotina de um mesmo analgésico para todas as crianças, independentemente de sua queixa. Isto leva ao questionamento se a tolerância à dor de cada criança está sendo efetivamente considerada, e também sobre sua capacidade de comunicação, já que muitas vezes as prescrições são atribuídas à condição 'se necessário'(12).

A Organização Mundial da Saúde tem estabelecido, como prioridade no atendimento a pacientes oncológicos, o alívio da dor. Pacientes pediátricos devem ser tratados com fármacos analgésicos e orientação psicológica para o manejo adequado da dor, objetivando, além de minimizar a dor, capacitar a criança e a família para entender o que está acontecendo. E, nesse sentido, criar métodos de avaliação e localização da dor conforme a faixa etária e desenvolvimento psicomotor 
e cognitivo de cada criança é de suma importância, buscando-se traduzir os dados subjetivos referidos na forma mais objetiva possível. Neste contexto, utilizamse protocolos em que todos os profissionais avaliam de forma sistemática a experiência dos pacientes. Isto é utilizado para criar parâmetros que permitam estabelecer protocolos de assistência na dor e a avaliação contínua quanto a sua eficácia, bem como para treinamento da equipe ${ }^{(13)}$.

Entretanto, estudos salientam a importância de se utilizar esquemas não farmacológicos para melhorar o conforto do paciente. São exemplos: relacionamento confiante; ambiente calmo; promoção de repouso e relaxamento; mudanças de posição; desvio da atenção da dor para outro foco, fornecendo outros impulsos sensoriais; alteração na condução do estímulo e diminuição de estímulos nocivos. Ainda, a estes se acrescenta a promoção de autoconfiança e confiança; estabelecimento de uma boa comunicação-empatia; apoio emocional e técnicas como massagens, uso de compressas quentes ou frias e técnicas psicológicas (hipnose, musicoterapia, relaxamento, terapias ocupacionais e técnicas bio-energéticas ${ }^{(13-14)}$.

A localização da dor é um problema que foi constatado nesse estudo, pois as crianças menores (lactentes e pré-escolares) se expressam por meio do choro e pouco conseguem descrever a dor que sentem. Assim, a equipe tem dificuldade em identificar o local exato da dor, fazendo-se presente, em muitos casos, somente o tratamento medicamentoso para toda e qualquer manifestação de choro. Métodos menos invasivos e/ou estratégias alternativas de manejo, tais como bolsa de água quente e/ou massagem, foram raros, segundo os registros em prontuário, neste estudo.

No manejo da dor, as técnicas psicológicas podem surtir efeito bastante eficaz, principalmente se observarmos que as cinco crianças que apresentaram um número elevado de queixas de dor ficaram internadas na unidade por mais de vinte dias. Pode-se considerar que uma internação mais longa reflete um quadro clínico menos estável e, possivelmente, maior chance de apresentar dor. No entanto, ressalta-se a ideia de alguns estudos que relacionam a dor ao maior tempo de internação, visto que esta, durante a hospitalização é frequente devido aos efeitos da hospitalização para a criança. Assim, ela é vista como uma dupla agressão, interna e externa a ela, na medida em que a doença é um mal físico de origem interna e os procedimentos utilizados para o seu tratamento são sentidos como uma agressão de origem externa ${ }^{(15)}$.
A investigação suscitou muitas reflexões sobre o manejo da dor nas crianças com câncer, a qual pode advir da doença, dos procedimentos diagnósticos, dos efeitos colaterais do tratamento e do estresse psicológico. E, apesar de influenciar sobremaneira a evolução do tratamento da criança internada, a dor parece ser pouco valorizada, subnotificada, tratada inadequadamente ou, até mesmo, não tratada. Isso se explica, talvez, pela ideia de que a dor é um resultado esperado de muitos procedimentos e que a prescrição de analgésicos é sua resolução; há a tendência de reduzir a dor a um indicador de distúrbio fisiológico, que deve ser suprimido por meio de medicamentos.

\section{CONCLUSÕES}

A identificação e localização da dor foi um problema observado, demonstrando-se a necessidade do estabelecimento de critérios para sua avaliação de acordo com a idade e a maturidade da criança.

Com relação às queixas e ao manejo da dor foi possível perceber que é frequente quando a criança apresenta um tipo de câncer que atinge o sistema nervoso (neuroblastoma) e o sistema sanguíneo/linfático e ósseo (leucemias). Eventos que causam desconforto generalizado, muitas vezes tornando-se a dor a própria doença.

Este fato reforça a necessidade de se estabelecer métodos de avaliação e localização da dor conforme a faixa etária e o desenvolvimento psicomotor e cognitivo de cada criança, buscando aliar o conhecimento técnico-científico à sensibilidade, interesse, compaixão e afetividade. Assim, salienta-se a importância da equipe de enfermagem buscar continuamente competências e habilidades no manejo da dor em criança oncológica, pois o alívio desta é um direito básico do ser humano, independentemente da faixa etária.

As limitações do estudo relacionaram-se à qualidade dos registros de enfermagem: letra ilegível, falta de anotações sobre a satisfação, ou não, da conduta realizada e anotações exíguas, bem como a falta delas. Assim, recomenda-se especial atenção à realização de registros legíveis e completos, que contenham dados como início da queixa, tipo, intensidade e localização da dor, frequência, duração, providência tomada e resultados obtidos.

Recomenda-se, ainda, o investimento em educação em serviço no sentido de capacitar a equipe para a realização de anamnese e exame físico das crianças durante a queixa de dor, e sistematização de avaliação da dor, 
além de disponibilidade de protocolos de tratamento farmacológico e não farmacológico.

\section{REFERÊNCIAS}

1. Reis RS, Santos MOS, Thuler LCS. Incidência de tumores pediátricos no Brasil. Rev Bras Cancerol. 2007;53(1):5-15.

2. Arruda IB, Paula JMSF, Silva RPL. Efeitos adversos da quimioterapia antineoplásica em crianças: o conhecimento dos acompanhantes. Cogitare Enferm. 2009;14(3):535-9.

3. Brasil. Ministério da Saúde (BR). Instituto Nacional do Câncer - INCA. O problema do câncer no Brasil. 1997 [acesso em 1 nov 2007]. Disponível: http://www.inca. gov.br/conteudo_view.asp?id=349.

4. Fundação Centro de Controle de Oncologia do Estado do Amazonas - FCECON. Tipos de câncer mais comuns em crianças. [Internet]. Amazonas. 2005 [acesso em 2 nov 2007]. Disponível: http://www.fcecon.am.gov.br/ programas_02.php?cod=5849350.

5. Campos S. Câncer e dor. [Internet]. Medicina avançada. 2005 [acesso em 2 nov 2007]. Disponível: http://www. drashirleydecampos.com.br/noticias/14094.

6. Botega FH, Fontana RT. A dor como quinto sinal vital: utilização da escala de avaliação por enfermeiros de um hospital geral. Texto \& Contexto Enferm. 2010;19(2): 283-90.

7. Smeltzer SC, Bare BG. Brunner \& Suddarth: tratado de enfermagem médico-cirúrgica. $10^{\mathrm{a}}$ ed. Rio de Janeiro: Guanabara Koogan; 2005. v. 1

8. Minayo MCSA. O desafio do conhecimento: pesquisa qualitativa em saúde. $11^{\mathrm{a}}$ ed. São Paulo: Hucitec; 2008.

9. Braga PE, Latorre MRDO, Curado MP. Câncer na infância: análise comparativa da incidência, mortalidade e sobrevida em Goiânia (Brasil) e outros países. Cad Saúde Pública. 2002;18(1):33-44.

10. Vernier ETN. O empoderamento de cuidadoras de crianças com necessidades especiais de saúde: interfaces com o cuidado de enfermagem [tese]. Rio de Janeiro (RJ): Universidade Federal do Rio de Janeiro; 2007.

11. Persegona KR, Zagonel IPS. Relação intersubjetiva do enfermeiro e a criança com dor pós-operatória. Esc
Anna Nery. [Internet] 2008;12(3) [acesso em 11 ago 2010]. Disponível: http://www.eean.ufrj.br/revista enf/20083/artigo\%204.pdf

12. Rossato LM, Angelo M. Utilizando instrumentos para avaliação da percepção de dor em pré-escolares face a procedimento doloroso. Rev Esc Enferm USP. 1999;33(3):236-9.

13. Rigotti MA, Ferreira AM. Intervenções de enfermagem ao paciente com dor. Arq Ciênc Saúde. [Internet] 2005;12(1) [acesso em 5 nov 2007]. Disponível: http:// www.cienciasdasaude.famerp.br/Vol-12-1/09\%20-\%20 $\mathrm{id} \% 20105 . p d f$

14. Menossi MJ, Lima RAG. A dor da criança e do adolescente: dimensões do seu cuidar. Rev Bras Enferm. 2004;57(2):178-82.

15. Silva DB, Pires MMS, Nassar SM. Câncer pediátrico: análise de um registro hospitalar. J Pediatr. 2002;78(5):409-14. 